\title{
EFFect of A High-InTENSITy Isometric Potentiating Warm-up on Bat Velocity
}

\author{
Sheryl L. Gilmore, ${ }^{1}$ Lorrie R. Brilla, ${ }^{2}$ David N. Suprak, ${ }^{2}$ Gordon R. Chalmers, ${ }^{2}$ and \\ DYlan T. DAHLQUisT ${ }^{2}$ \\ ${ }^{1}$ Athletics Department, Edmonds Community College, Edmonds, Washington; and ${ }^{2}$ Biomechanics Laboratory, Department of \\ Health and Human Development, Western Washington University, Bellingham, Washington
}

\begin{abstract}
Gilmore, SL, Brilla, LR, Suprak, DN, Chalmers, GR, and Dahlquist, DT. Effect of a high-intensity isometric potentiating warm-up on bat velocity. J Strength Cond Res 33(1): 152$158,2019-T h i s$ study examined the acute effect of a highintensity isometric potentiating warm-up on subsequent maximal horizontal bat velocity in experienced female softball players $(n=28)$. The isometric potentiating warm-up consisted of 3 sets of 5 -second maximal voluntary contractions held in the early swing phase position, pulling against an immovable device. As one of the simplest methods to use the postactivation potentiation (PAP) stimulus, the warm-up was designed to acutely enhance muscle performance by inducing PAP. Because optimal recovery duration after a potentiating warmup can be highly variable, swing trials were conducted at predetermined rest intervals $(1,2,4,6,8,10$, and 12 minutes) to identify the recovery time that may have allowed for maximal possible benefits. Bat velocity was measured immediately before bat-ball impact using MaxTRAQ motion analysis software. The results showed that maximal horizontal bat velocity was significantly enhanced 6 minutes after the isometric warmup protocol (+1.27 m·s $\mathrm{s}^{-1},+2.84 \mathrm{mph},+4.93 \%$; $\left.p<0.05\right)$. In experienced female softball athletes, a specific isometric warmup may acutely enhance maximal horizontal bat velocity.
\end{abstract}

KEY WoRDS postactivation potentiation, female, softball, athletes

Address correspondence to Lorrie R. Brilla, lorrie.brilla@wwu.edu. $33(1) / 152-158$

Journal of Strength and Conditioning Research

Copyright (c) 2018 The Author(s). Published by Wolters Kluwer Health, Inc. on behalf of the National Strength and Conditioning Association. This is an openaccess article distributed under the terms of the Creative Commons Attribution-Non Commercial-No Derivatives License 4.0 (CCBY-NC-ND), where it is permissible to download and share the work provided it is properly cited. The work cannot be changed in any way or used commercially without permission from the journal.

\section{INTRODUCTION}

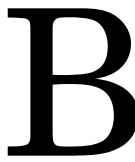

at velocity is one of the most critical factors for successful offensive performance in fastpitch softball. Softball players must possess both proper swing mechanics and the ability to optimize explosive rotational force production in a short amount of time (26). Although the research specifically regarding softball is insufficient, various baseball products and warm-up protocols have been developed with the goal of acutely enhancing bat velocity (3). As college softball hitters actually have less time to react to a pitched ball than many professional baseball players (3), any exercise or warm-up activity that leads to increased bat velocity in female softball players may have positive implications.

Postactivation potentiation (PAP) has recently become an increasingly popular method to increase power performance. Postactivation potentiation is the enhancement of muscle function after a high-force activity. After a short bout of high-intensity exercise, or a preload stimulus, the muscles involved are both fatigued and potentiated (16). Postactivation potentiation supports the notion of alternating heavy and light resistances to increase power output (8). As one of the simplest methods to use a PAP stimulus, weighted implements are often added to baseball and softball bats during a prebatting warm-up routine to optimize at-bat bat velocity (17); however, insufficient data exist to support the efficacy of these devices. Although a perceived increase in bat speed exists after a weighted bat warm-up, multiple studies show that using weighted bats elicits no significant difference in bat speed production in both baseball and softball players $(17,26,27)$. In other research, a decrement in bat speed has been found after using weighted bats $(2,22)$.

New research, however, has indicated that high-intensity isometric muscle actions can evoke a greater muscle PAP than dynamic conditions (15). As recommended in the research, this type of warm-up and training may improve rate of force development and increase power performance (28). Therefore, the purpose of this study was to determine the acute effect of a high-intensity isometric potentiating warm-up on subsequent maximal bat velocity in experienced female softball players. 
TABLE 1. Subject characteristics. ${ }^{*} \dagger$

\begin{tabular}{lcc}
\hline & Mean & $\pm S D$ \\
\hline Subject age (y) & 20 & 2.6 \\
Subject experience (y) & 11.5 & 3.2 \\
Subject height (cm) & 162 & 29 \\
Subject body mass (kg) & 69.19 & 11.36 \\
ABS (1RM bench press) (kg) & 43.44 & 6.95 \\
ABS (1RM back squat) (kg) & 75.18 & 11.71 \\
REL (ABS bench press/BM) (\%) & 0.63 & 0.09 \\
REL (ABS back squat/BM) (\%) & 1.10 & 0.17 \\
\hline
\end{tabular}

$* 1 \mathrm{RM}=1$ repetition maximum.

$\dagger$ Absolute strength (ABS) refers to muscular strength not related to body mass. Relative strength (REL) refers to strength per kilogram body mass.

\section{Methods}

\section{Experimental Approach to the Problem}

The experimental hypothesis states that the potentiating warm-up using high-intensity isometric contractions will elicit a significant increase in bat velocity when compared with subjects' established baseline bat velocity.

\section{Subjects}

A single group repeated measures to analyze the effect of a potentiating warm-up on maximal horizontal bat velocity. Twenty-eight $(\mathrm{n}=28)$ female subjects, aged 16-25 $(20 \pm 2.6)$ years old, volunteered for this study. All subjects were healthy, competitive fastpitch softball players with an average of 11.5 $( \pm 3.2)$ years of experience. Mean values and $S D$ s for subject

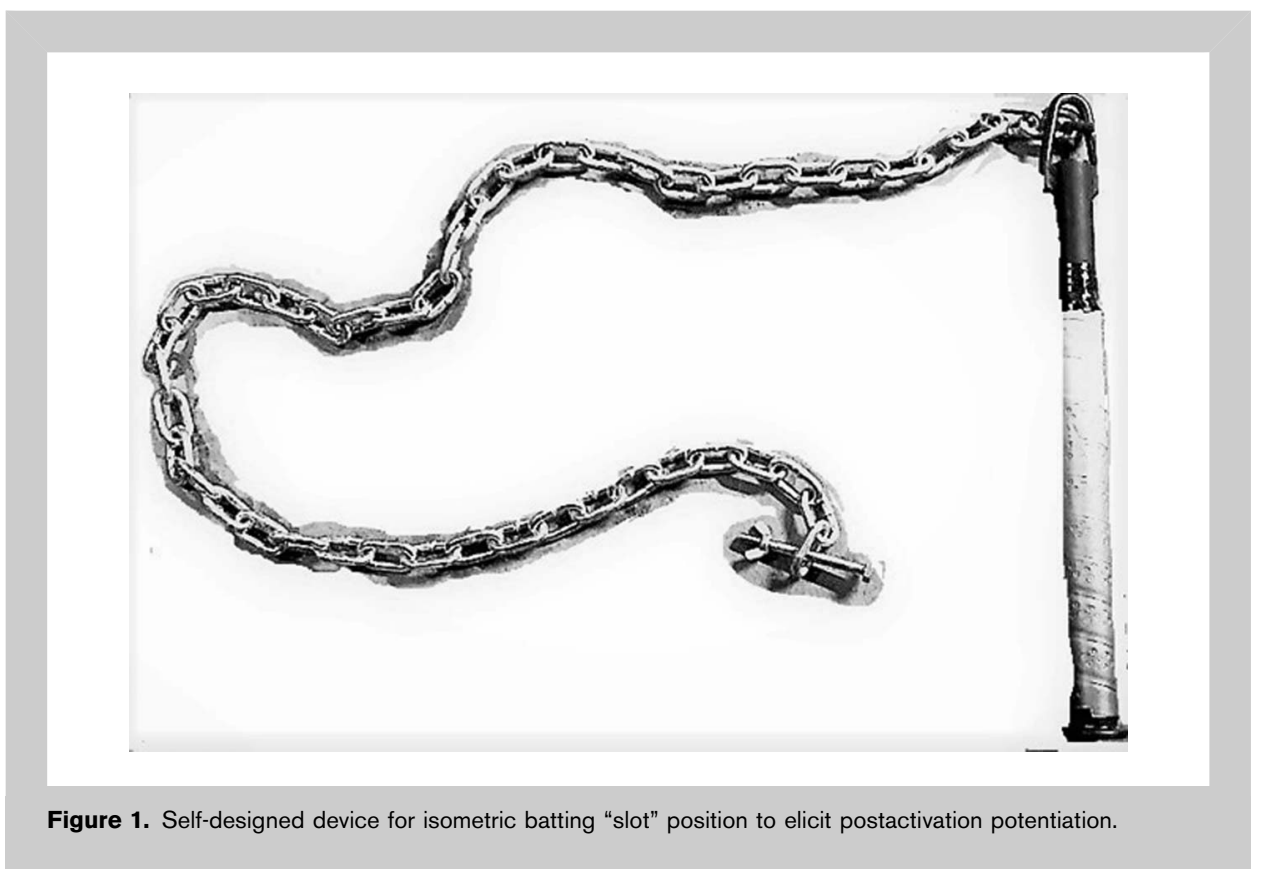

characteristics, including absolute strength (ABS) and relative strength (REL), are provided in Table 1 . All experimental procedures were approved by the human subjects review board at Western Washington University. The risks and benefits of participation in this study were explained to each subject and their parent(s) or guardian(s) (if the subject was younger than 18 years). After this, subjects completed written informed consent and parents and guardians also provided written informed consent for subjects under the age of 18 and a permission to videotape form before partaking in the study.

\section{Procedures}

The isometric potentiating protocol used a device designed by the lead author (Figure 1). The device consisted of a softball bat handle, wrapped with bat grip tape, attached to a chain, 102 centimeters in length, which in turn attached to a height adjustable fitting on a wall. Horizontal bat velocity was measured during each swing trial using a Casio $(\mathrm{Ca}-$ sio Computer Co., Ltd., Tokyo, Japan) high-speed video camera, filming at $420 \mathrm{~Hz}$.

The camera was positioned on a tripod, perpendicular to the hitting zone, in the frontal plane. A meter stick aligned in the field of view of the video camera served as a distance reference, which was used to calculate bat displacement per video frame. Bright reflective tape was fastened to the barrel of a Louisville Slugger Catalyst fastpitch bat (Louisville Slugger, Louisville, KY, USA) of standard length, 83.8 centimeters (33 inches), and weight, 23 ounces. This tape served as a marker during filming to make the manual digitization consistent throughout the trials. As recommended by the research (29), bat velocity was measured immediately before ball contact. The distance that the reflective marker on the bat barrel traveled in each frame of the high-speed video recording was later used to determine bat velocity. The video position data was analyzed using MaxTRAQ motion analysis software (Innovision Systems, Inc., Columbiaville, MI, USA) and later exported into Excel 2010 (Microsoft Corp., Bellevue, WA, USA) for further analysis.

Measurement Techniques and Testing Procedures. Inclusion for this study demanded that subjects be free of any musculoskeletal or neurological impairment or injury. Subjects abstained from any heavy resistance exercise and creatine supplementation 48 hours before testing sessions. Subjects were also to refrain from consuming alcohol (24 hours) and caffeine ( 8 hours) before the experiments. 
TABle 2. Mean, $S D$, and change score data for maximal horizontal bat velocity during the preand post-warm-up swing trials.*

\begin{tabular}{|c|c|c|c|c|}
\hline \multirow[b]{2}{*}{ Time } & \multicolumn{2}{|c|}{ Bat velocity } & \multicolumn{2}{|c|}{$\begin{array}{l}\text { Change in bat velocity } \\
\text { from baseline }\end{array}$} \\
\hline & $\left(m \cdot s^{-1}\right)$ & $\pm S D$ & $\left(m \cdot s^{-1}\right)$ & $\%$ \\
\hline Baseline & 25.74 & 2.65 & N/A & N/A \\
\hline Post 1 min & 25.46 & 3.05 & -0.28 & -1.09 \\
\hline Post 2 min & 25.98 & 2.99 & 0.24 & 0.94 \\
\hline Post 4 min & 26.06 & 3.00 & 0.32 & 1.26 \\
\hline Post 6 min & $27.01 \dagger t$ & 2.97 & 1.27 & 4.93 \\
\hline Post 8 min & 26.54 & 3.05 & 0.80 & 3.12 \\
\hline Post $10 \mathrm{~min}$ & 26.31 & 2.65 & 0.57 & 2.23 \\
\hline Post $12 \mathrm{~min}$ & 26.12 & 3.11 & 0.48 & 1.49 \\
\hline
\end{tabular}

*Percent change (\%) represents the change from baseline bat velocity $\left(25.74 \pm 2.65 \mathrm{~m} \cdot \mathrm{s}^{-1}\right)$.

$\dagger$ The results are significantly different from baseline bat velocity $(p<0.05)$.

$\ddagger$ The results are significantly different from 1-minute post-warm-up bat velocity $(p<0.05)$.

Predictive one repetition maximum (1RM) using multiple repetition (repetitions to fatigue) testing was selected for this study as described elsewhere (13). This method is reliable, safe, and accurate when using the equation: $1 \mathrm{RM}=(0.025$ $\times$ [repetition weight $\times$ repetitions to failure] $)+$ repetition weight to predict $1 \mathrm{RM}$ strength for the bench press and back squat. The results were then used to determine ABS and REL.

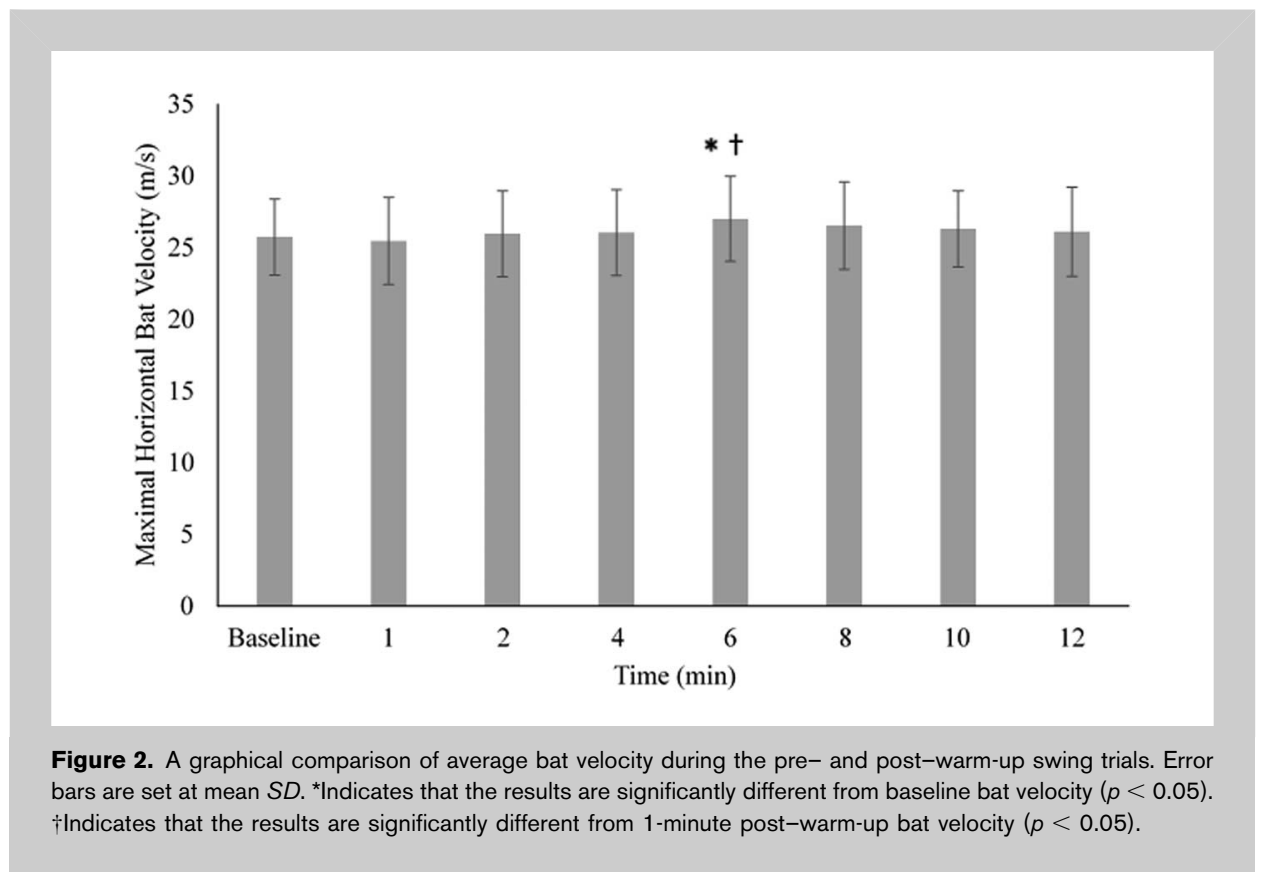

Subjects began a standardized warm-up consisting of general, dynamic, and specific phases. The general warmup consisted of jogging on a treadmill for 2 minutes at 6 miles per hour. Immediately after the general warm-up, subjects completed a dynamic warm-up, which followed their typical pregame dynamic stretching routine. After a 4minute rest period, the subjects completed a specific warmup consisting of 1 set of 5 dry swings (practice swings that do not involve making contact with the ball), increasing in intensity, with the testing bat. The dry swings were followed by 1 set of 5 warm-up swings hitting a teed ball into a net. The last 3 swings were to be performed at maximal effort. The tee was positioned down the center and at the forward edge of home plate. Tee height was set even with each hitter's umbilicus during the blocking, or middle, phase of the swing. To increase the consistency of the swings throughout the swing trials, tape was placed on the ground marking comfortable foot placement for the subject during the teed warm-up swings. The balls used in testing were 12 inches, game-ball yellow, Lite-Flite softballs (Jugs Sports, Tualatin, OR, USA). During a 2-minute recovery period, the high-speed camera was set into position to fully capture the subject's swing. After the 2-minute recovery, subjects were told to assume a stance and swing as if they were in a game situation. Rest periods of 20 seconds were given between swings to recover and simulate the time taken between pitches in a game situation. Given that horizontal velocity of the bat head was being measured, data may have been affected by different aspects of the swing, such as the angle and location of bat-ball impact. To account for this, subjects were asked to drive the ball on a line up the middle toward what would be centerfield. A red square $(30 \times 30$ $\mathrm{cm})$ was placed in the center of the net as a visual target for all subjects to hit. The researcher, who had intercollegiate softball experience, made a qualitative judgment as to whether or not each swing was successful and met a qualitative analysis of proper bat mechanics (19). Three maximal swings, which met the requirements, were recorded. The average velocity of the 3 swings was used for analysis and considered to be the subject's baseline bat velocity.

Once baseline bat velocity was established, subjects were given 10 minutes to recover. During this time, clear instructions regarding the phasespecific isometric conditioning contraction were given to each subject (described below). The 


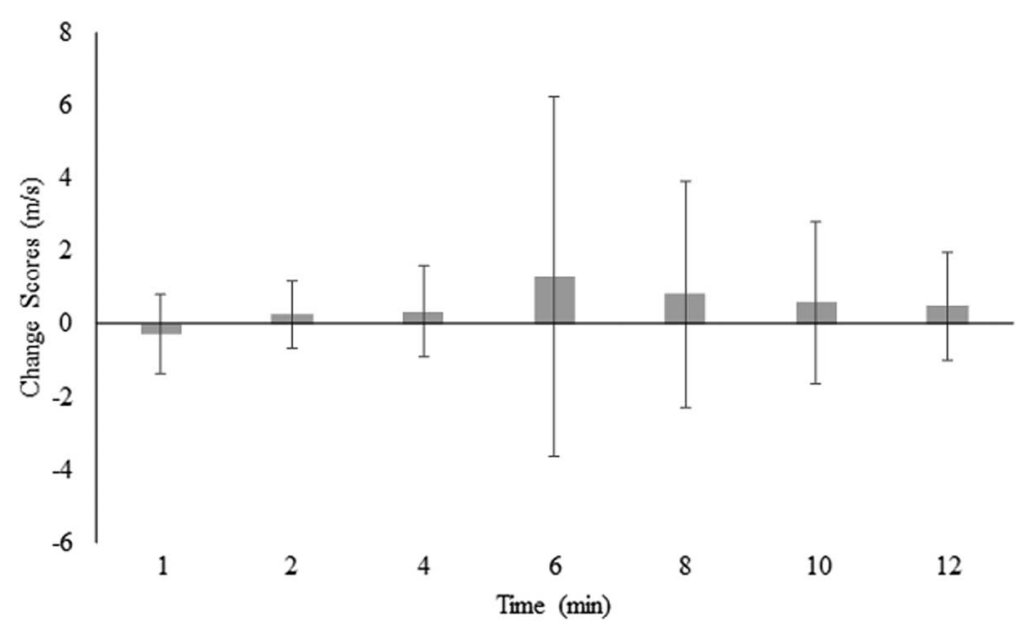

Figure 3. A graphical comparison of average change in bat velocity between baseline and the post-warm-up swing trials. experimental potentiating isometric warm-up consisted of each subject pulling against a bat handle connected to a chain attached to a wall. Subjects were required to elicit maximal voluntary contractions (MVCs) as if attempting to break free and finish their swing. Three maximal effort isometric contractions were sustained in this position for 5 seconds. Each isometric MVC was separated by 30 seconds of recovery. All subjects were instructed to slowly progress through their swing motion, initiated in the legs, and stop in the early swing phase, approaching the "slot" position (elbow and knee of the trailing arm and leg are aligned vertically, and the hips are neither fully closed nor fully open), which occurs during the early to middle phase of the swing. This "slot" position is believed to be a critical point in the swing for muscle activation and the efficient transfer of force (22). While in this position, the chain (connected to the bat handle and the wall) was stretched tight, and any further movement forward in the swing was prohibited by the chain. The main criterion was that the elbow and knee of the trailing arm and leg were aligned vertically and that the hips were neither fully closed nor fully open. To ensure that each subject was properly activating the muscles involved in this position (trailing leg muscles, trunk muscles, and lead arm), the researcher performed manual muscle tests on the trail leg biceps femoris and gluteus maximus, the abdominal obliques, and the lead arm posterior deltoid and triceps during each contraction. The researcher felt for very firm contraction in the target muscle being examined to verify its activation. Manual palpation was used instead of muscle activation measured by electromyography to mimic what would be expected in a field situation.

On completion of the potentiating protocol, each subject returned to the batter's box and was instructed to swing the bat maximally at the teed softballs. Trials were taken at 1,2 , $4,6,8,10$, and 12 minutes after exercise. All trials were recorded with the high-speed video camera. All trials were imported into MaxTRAQ motion analysis software (Innovision Systems, Inc.) and manually digitized by the researcher. Each video was cut down to the range of interest, which began just before each subject reached slot position and ended just after ball contact. The sweet spot of the bat, identified by the bright reflective marker, was digitized for every frame in the range of interest. Once digitization was complete, the position data were exported from MaxTRAQ into Excel 2010 (Microsoft Corp.). Bat velocity was calculated from the position data and the frame rate, using the first central difference method. Maximal horizontal bat velocity was then established by finding the maximum value.

\section{Statistical Analyses}

A one-way repeated-measures analysis of variance was used to examine the effect of time (baseline vs. 1, 2, 4, 6, 8, 10, and 12 minutes after isometric warm-up) on peak bat velocity with SPSS (Version 25, Chicago, IL, USA). Mean values \pm $S D$ s were calculated for baseline peak bat velocity and peak bat velocity at each of the 7 time points after the potentiating warm-up protocol. In the event of a significant effect, post hoc testing using pairwise comparisons, with a Bonferroni correction, was conducted. A polynomial trend analysis was also completed, as well as tests for effect size. Change scores were calculated for each time point after the potentiating warm-up protocol. The following equation was used: change score $=$ potentiated variable - unpotentiated variable. The change in velocity was also expressed as a percentage change using the equation: change score $\%=$ ([potentiated unpotentiated]/unpotentiated) $\times 100$.

\section{Results}

There was a significant effect of the isometric potentiating warm-up protocol on bat velocity over time $(p<0.001)$, presented in Table 2. The effect size is medium $\left(\omega^{2}=\right.$ $0.143)$. Post hoc testing revealed that bat velocity was significantly higher 6 minutes after the potentiating protocol when compared with baseline bat velocity $(p<0.05)$ and bat velocity at 1 minute after the potentiating protocol $(p<0.05)$ (Figure 2). The results also indicate that there is a significant quadratic trend, with bat velocity peaking at 
6 minutes and subsequently decreasing $(p<0.05)$. The effect size for this trend is large $\left(\omega^{2}=0.246\right)$.

Subjects exhibited potentiation at all time points after the isometric warm-up, with the exception of the 1-minute swing trial. However, maximal horizontal bat velocity was only significantly potentiated 6 minutes after the highintensity isometric potentiating warm-up, eliciting an average increase in bat velocity of $1.27 \mathrm{~m} \cdot \mathrm{s}^{-1}(2.84 \mathrm{mph})$ when compared with baseline bat velocity (Figure 3 ).

\section{Discussion}

The current study investigated the acute effect of a highintensity isometric potentiating warm-up on subsequent maximal bat velocity in experienced female softball players. The findings indicated maximal horizontal bat velocity can be significantly augmented 6 minutes after a phase-specific high-intensity isometric warm-up protocol, consisting of three 5-second isometric contractions at maximal effort, in experienced high school and collegiate softball players. Furthermore, bat velocity was elevated above baseline at 2 , $4,6,8,10$, and 12 minutes after the conditioning contractions, displaying a window of potentiation between 2 and 12 minutes of recovery. Optimal recovery time may shift, depending on the individual. A significant quadratic trend, peaking at 6 minutes, indicates that the subjects involved in this study followed a general pattern of increasing and then decreasing their bat velocity over time after the potentiating protocol. The positive effect of the potentiating warm-up protocol, as well as the time frame of potentiation, is similar to what has been reported in the literature regarding PAP and explosive performance $(4,9,16,28)$.

The similarity between the current study and the literature demonstrates PAP may be due to the close adherence to the load (maximal), volume (multiple sets), and recovery time (4-12 minutes) in the methods, whereas the difference in percent potentiation may be due to the differences in the potentiated activity, as well as the sex and level of training of the subjects. The studies reporting $5-10 \%$ increases in peak power output tested activities, such as the countermovement jump and explosive bench press throw, and used elite male athletes, such as professional rugby players $(9,16)$. Thus, well-trained individuals may have a greater capacity for potentiation $(9,15,16)$ when compared with the population used in the current study.

In relationship to this study, Higuchi et al. (6) examined the acute change in bat velocity after 3 types of warm-up procedures using National Collegiate Athletic Association (NCAA) Division I collegiate baseball players. The 3 types of prebatting warm-ups consisted of (1) 5 standard bat swings (1), 5 weighted bat swings, and (2) 4 sets of 5second maximal voluntary isometric contractions mimicking the bat swing movement pattern (6). For the isometric stimulus, the researchers used single hand pulls (lead hand in set 1 and 3 , trail hand in sets 2 and 4). The results indicated that after 1 minute of recovery, the standard bat warm-up did not significantly change bat velocity $\left(-0.33 \mathrm{~m} \cdot \mathrm{s}^{-1}\right)$, and the weighted bat warm-up significantly decreased bat velocity $\left(-0.89 \mathrm{~m} \cdot \mathrm{s}^{-1}\right)$. However, the maximal isometric warm-up significantly increased bat velocity $\left(+0.39 \mathrm{~m} \cdot \mathrm{s}^{-1}\right)(6)$. The authors did not report bat velocity at any other time point.

Unlike the findings of Higuchi et al. (6), the current study showed that bat velocity decreased after 1 minute of recovery. However, an increase in bat velocity, similar to what Higuchi et al. (6) reported, was found 4 minutes after the isometric warm-up protocol. The difference in the window of potentiation may be due to possible differences in muscle mass and strength between the Division I male baseball players compared with the female high school and collegiate softball players $(20,21)$. Because women have on average lower percent of lean muscle tissue compared with male counterparts (11) confirmed with the low REL in the current study results, the intense conditioning contractions may more easily deplete the muscle phosphocreatine (PCr) stores and consequently induce greater fatigue in the softball players, when compared with high-level baseball players. Therefore, in softball players, the window for potentiation may occur later, after fatigue subsides. However, it is important to note that some fatigue is expected and that the experienced female softball players only exhibited 1 minute of decreased performance after the isometric warm-up. Highlevel baseball players may have a greater capacity for potentiation, hypothetically making the isometric warm-up more effective, suggesting that PAP manifests to a greater degree in athletes with high relative strength (24).

Performance after a potentiating protocol depends on the interplay between fatigue, which impairs performance, and potentiation, which enhances performance (17). The depletion of PCr may be responsible for the fatigue experienced after a conditioning contraction (7). In order for a potentiating warm-up protocol to be effective in acutely enhancing muscle performance, the phosphorylation of myosin regulatory light chains (RLCs) must be able to outlast the time needed for repletion of phosphagen stores in the muscle (28). Resynthesis of PCr stores usually occurs between 4 and 8 minutes after exercise $(1,5)$, while the time course for the rate of dephosphorylation of myosin RLCs through the slow activity of myosin light chain phosphatase seems to be around 12 minutes (12). With this information, a window of potentiation for optimal performance may exist between 4 and 12 minutes of recovery. The time course of potentiation found in this study closely resembles what is presented above, providing putative mechanisms for the quadratic trend observed; with performance increasing after 2 minutes of recovery, peaking at 6 minutes, and decreasing as 12 minutes of recovery approaches. The peak potentiation at 6 minutes, in this study, occurred after the measures at 1 , 2 , and 4 minutes after warm-up, and these experimental measures are expected to contribute to the combination of fatigue and recovery the subjects experienced. In a game situation, in which there may not be evenly spaced repeated 
maximal swing trials between the warm-up and the at-plate performance, the timing of the peak potentiation may vary.

Based on the literature and the findings presented here, a high-intensity isometric potentiating warm-up may enhance maximal horizontal bat velocity in female softball players through the mechanism of PAP. The ability of a baseball or softball player to increase maximal horizontal bat velocity provides more time to observe the oncoming pitch, allowing for a more accurate evaluation of the speed, location, and movement of the ball, increasing the hitter's chances of making solid contact (18). Increased bat velocity also facilitates increased batted ball distance and velocity $(2,25)$. According to previous research, the widely used weighted bat warm-up produces no effect or a negative effect on post-warm-up bat velocity $(2,17,23,26,27)$. Furthermore, the after effects of swinging a weighted bat with an increased moment of inertia can not only change a batter's perception of the swing (14), but also the batter's swing pattern $(10,23)$. Instead, using a high-intensity isometric prebatting warm-up protocol can be a positive alternative to optimally increase at-bat bat velocity.

\section{Practical Applications}

The effect of isometric exercise on PAP, established in multiple studies, has profound implications for trainers, coaches, athletes, and fitness enthusiasts. Performing maximal or near MVC, pushing or pulling against fixed objects, could be a very simple and cost-effective way to produce a state of PAP before sports performance or training sessions that require high force and power outputs. The timing of PAP in this given situation is crucial and could be a limitation to apply the results of this study for the first batter to plate in an inning, although all following batters may not have this problem.

Although this is not always the case, on average, a batter will spend $\sim 2$ minutes on-deck warming up (30). If it takes a minimum of 1 minute to recover from this PAP exercise (as shown by our results from this study), a batter could be able to effectively implement this protocol. Given the total amount of time, batters would step up to plate within the possible optimal time zone ( $\sim 6$ minutes). As previously reported in a study on various warm-up devices (31), the bat velocity enhancement in the current study falls within the time window (4-12 minutes) of when the greatest effect would be elicited if the stimulus is applied in the on-deck period.

The current results show that the bat velocity enhancement occurred over a range from 4 to 12 minutes, peaking at 6 minutes. Although it is not possible to predict the exact time in the future when a player will reach the plate in a game situation, it is feasible that a player could predict that it is likely that they will be at bat in the next 5-13 minutes and so start the 1 minute 15 seconds of preparation procedure. If they subsequently end up batting 4-12 minutes after the preparation, the data suggest that their swing velocity will be enhanced.
The key findings of this study were that PAP could be elicited in female softball players and that the timing of the performance enhancements may be appropriate for application to game situations. The results imply that this sportspecific potentiating warm-up may be effective in enhancing offensive performance in experienced female high school and college softball players by significantly increasing maximal horizontal bat velocity.

\section{Acknowledgments}

The authors thank the student-athletes who participated in this study. All authors declare no conflict of interest. The results of this study do not constitute endorsement of the product by the authors or the National Strength and Conditioning Association.

\section{REFERENCES}

1. Bassett, KE, Szymanski, DJ, Beiser, EJ, Till, ME, Medlin, GL, and DeRenne, C. Effects of various warm-up devices on bat swing velocity of college softball players. J Strength Cond Res 25: S71, 2011.

2. DeRenne, C, Ho, KW, Hetzler, RK, and Chai, DX. Effects of warm up with various weighted implements on baseball bat swing velocity. J Appl Sport Sci Res 6: 214-218, 1992.

3. Flyger, N, Button, C, and Rishiraj, N. The science of softball: Implications for performance and injury prevention. Sports Med 36: 797-816, 2006.

4. Gullich, A and Schmidtbleicher, D. MVC-induced short-term potentiation of explosive force. New Stud Athletics 4: 67-81, 1996.

5. Harris, RC, Edwards, RH, Hultman, E, Nordesjö, LO, Nylind, B, and Sahlin, K. The time course of phosphorylcreatine resynthesis during recovery of the quadriceps muscle in man. Pflugers Archiv 367: 137-142, 1967.

6. Higuchi, T, Nagami, T, Mizuguchi, N, and Anderson, T. The acute and chronic effects of isometric contraction conditioning on baseball bat velocity. J Strength Cond Res 27: 216-222, 2013.

7. Hirvonen, J, Nummela, A, Rusko, H, Rehunen, S, and Härkönen, M. Fatigue and changes of ATP, creatine phosphate, and lactate during the 400-m sprint. Can J Sport Sci 17: 141-144, 1992.

8. Hodgson, M, Docherty, D, and Robbins, D. Post-activation potentiation: Underlying physiology and implications for motor performance. Sports Med 35: 585-595, 2005.

9. Kilduff, LP, Bevan, HR, Kingsley, MI, Owen, NJ, Bennett, MA, Bunce, PJ, et al. Postactivation potentiation in professional rugby players: Optimal recovery. J Strength Cond Res 21: 1134-1138, 2007.

10. Liu, C, Liu, YC, Kao, YC, and Shiang, TY. Effects of training with dynamic moment of inertia bat on swing performance. $J$ Strength Cond Res 25: 2999-3005, 2011.

11. Miller, AE, MacDougall, JD, Tarnopolsky, MA, and Sale, DG. Gender differences in strength and muscle fiber characteristics. Eur J Appl Physiol Occup Physiol 66: 254-262, 1993.

12. Moore, RL and Stull, JT. Myosin light chain phosphorylation in fast and slow skeletal muscles in situ. Amer J Physiol 247: C462-C471, 1984.

13. O'Connor, B, Simmons, J, and O'Shea, P. Weight Training Today (Vol. 1). St. Paul, MN: West Publishing, 1989. pp. 1-135.

14. Otsuji, T, Abe, M, and Kinoshita, H. After-effects of using a weighted bat on subsequent swing velocity and batters' perceptions of swing velocity and heaviness. Percept Mot Skills 94: 119-126, 2002.

15. Race, DM. A cinematographic and mechanical analysis of the external movements involved in hitting a baseball effectively. Res $Q$ 1961;32: 394-404. 
16. Rassier, DE and Macintosh, BR. Coexistence of potentiation and fatigue in skeletal muscle. Bra J Med Bio Res 33: 499-508, 2000.

17. Reyes, GF and Dolny, DG. Acute effects of various weighted bat warm-up protocols on bat velocity. J Strength Cond Res 23: 21142118, 2009.

18. Reyes, GF, Dickin, DC, Dolny, DG, and Crusat, JK. Effects of muscular strength, exercise order, and acute whole-body vibration exposure on bat swing speed. J Strength Cond Res 24: 3234-3240, 2010.

19. Rixon, KP, Lamont, HS, and Bemben, MG. Influence of type of muscle contraction, gender, and lifting experience on postactivation potentiation performance. J Strength Cond Res 21: 500-505, 2007.

20. Seitz, LB, Villarreal, ES, and Haff, GG. The temporal profile of postactivation potentiation is related to strength level. J Strength Cond Res 28: 706-715, 2014.

21. Seitz, LB, Trajano, GS, Haff, GG, Dumke, CC, Tufano, JJ, and Blazevich, AJ. Relationships between maximal strength, muscle size, myosin heavy chain isoform composition and post-activation potentiation. Appl Physiol Nutr Metab 41: 491-497, 2016.

22. Shaffer, B, Jobe, FW, Pink, M, and Perry, J. Baseball batting: An electromyographic study. Clin Ortho Relat Res 292: 285-293, 1993.

23. Southard, D and Groomer, L. Warm-up with baseball bats of varying moments of inertial: Effect on bat velocity swing pattern. Res Q Ex Sport 74: 270-276, 2003.

24. Sygulla, KS and Fountaine, CJ. Acute post-activation potentiation in NCAA Division II female athletes. Int J Exerc Sci 7: 212-219, 2014.
25. Szymanski, DJ, McIntyre, JS, Szymanski, JM, Bradford, JT, Schade, $\mathrm{RL}$, Madsen, $\mathrm{NH}$, et al. Effect of torso rotational strength on angular hip, angular shoulder, and linear bat velocities of high school baseball players. J Strength Cond Res 21: 1117-1125, 2007.

26. Szymanski, DJ, DeRenne, C, and Spaniol, FJ. Contributing factors for increased bat swing velocity. J Strength Cond Res 23: 1338-1352, 2009.

27. Szymanski, DJ, Beiser, EJ, Bassett, KE, Till, ME, Medlin, GL, Beam, $\mathrm{JR}$, et al. Effect of various warm-up devices on bat velocity of intercollegiate baseball players. J Strength Cond Res 25: 287-292, 2011.

28. Tillin, NA and Bishop, D. Factors modulating post-activation potentiation and its effect on performance of subsequent explosive activities. Sports Med 39: 147-166, 2009.

29. Welch, CM, Banks, SA, Cook, FF, and Draovitch, P. Hitting a baseball: A biomechanical description. J Ortho Sports Phy Ther 22: 193-201, 1995.

30. Williams, CC, Gdovin, JR, Wilson, SJ, Cazas-Moreno, VL, Eason, JD, Hoke, EL, et al. The effects of various weighted implements on baseball swing kinematics in collegiate baseball players. J Strength Cond Res 2017. Epub ahead of print.

31. Wilson, JM, Miller, AL, Szymanski, DJ, Duncan, NM, Andersen, JC, Alcantara, ZG, et al. Effects of various warm-up devices and rest period lengths on batting velocity and acceleration of intercollegiate baseball players. J Strength Cond Res 26: 2317-2323, 2012. 